\title{
PRMT5 inhibition attenuates cartilage degradation by reducing MAPK and NF-KB signaling
}

Yonghui Dong ${ }^{1}$, Ping Wang ${ }^{2,3}$, Yongguang Yang ${ }^{1}$, Jincheng Huang ${ }^{1}$, Zhipeng Dai ${ }^{1}$, Wendi Zheng ${ }^{1}$, Zhen Li ${ }^{1}$, Zheng Yao ${ }^{1}$, Hongjun Zhang ${ }^{1}$ and Jia Zheng ${ }^{1 *}$ (D)

\begin{abstract}
Objectives: A role for the type II arginine methyltransferase PRMT5 in various human diseases has been identified. In this study, the potential mechanism underlying the involvement of PRMT5 in the pathological process leading to osteoarthritis (OA) was investigated.

Methods: PRMT5 expression in cartilage tissues from patients with OA and control individuals was assessed by immunohistochemical staining. The regulatory and functional roles of PRMT5 in the chondrocytes of patients with $\mathrm{OA}$ and control individuals were determined by western blotting and reverse transcription polymerase chain reaction. The effects of the PRMT5 inhibitor EPZ on interleukin-1 $\beta$-induced inflammation were examined in the chondrocytes of patients with OA and in the destabilized medial meniscus (DMM) of a mouse model of OA.

Results: PRMT5 was specifically upregulated in the cartilage of patients with OA. Moreover, adenovirus-mediated overexpression of PRMT5 in human chondrocytes caused cartilage degeneration. This degeneration was induced by elevated expression levels of matrix-degrading enzymes (matrix metalloproteinase-3 (MMP-3) and matrix metalloproteinase13 (MMP-13)) in chondrocytes. The activation of the MAPK and nuclear factor KB signaling pathways was evidenced by elevated levels of $\mathrm{p}-\mathrm{p} 65, \mathrm{p}-\mathrm{p} 38$, and $\mathrm{p}$-JNK. These effects were attenuated by inhibiting the expression of PRMT5. In the mouse model, EPZ inhibited PRMT5 expression, thus protecting mouse cartilage from DMM-induced OA.
\end{abstract}

Conclusions: Our results demonstrate that PRMT5 is a crucial regulator of OA pathogenesis, implying that EPZ has therapeutic value in the treatment of this cartilage-destroying disease.

Keywords: Osteoarthritis, PRMT5, Chondrocytes, Kinases, DMM model

\section{Introduction}

Osteoarthritis (OA) is a whole-joint disease characterized by chronic joint pain and loss of function, which occurs in $50 \%$ of the population $\geq 65$ years of age $[1,2]$. The development of OA includes degeneration of the articular cartilage, induction of a synovial inflammatory

\footnotetext{
* Correspondence: zhengjia90180@sina.com

'Department of Orthopedics, Henan Provincial People's Hospital, Zhengzhou University People's Hospital, Henan University People's Hospital, No.7, Weiwu Road, Zhengzhou 450003, Henan Province, China

Full list of author information is available at the end of the article
}

response, formation of osteophytes, and remodeling of the subchondral bone [3]. Among these pathological changes, cartilage degeneration is the key feature of $\mathrm{OA}$; this is caused by activation of matrix-degrading enzymes, such as matrix metalloproteinase-3 (MMP-3) and matrix metalloproteinase-13 (MMP-13) [4-6]. Both enzymes are regulated by inflammatory cytokines (e.g., tumor necrosis factor $\alpha$ and interleukin (IL)-1 $\beta$ ), which are released by chondrocytes $[7,8]$ and induce the production of MMPs by activating the nuclear factor (NF) $-\mathrm{kB}$ and mitogen-activated protein kinase (MAPK) cellular signaling

C C The Author(s). 2020 Open Access This article is licensed under a Creative Commons Attribution 4.0 International License, which permits use, sharing, adaptation, distribution and reproduction in any medium or format, as long as you give appropriate credit to the original author(s) and the source, provide a link to the Creative Commons licence, and indicate if changes were made. The images or other third party material in this article are included in the article's Creative Commons licence, unless indicated otherwise in a credit line to the material. If material is not included in the article's Creative Commons licence and your intended use is not permitted by statutory regulation or exceeds the permitted use, you will need to obtain permission directly from the copyright holder. To view a copy of this licence, visit http://creativecommons.org/licenses/by/4.0/ The Creative Commons Public Domain Dedication waiver (http://creativecommons.org/publicdomain/zero/1.0/) applies to the data made available in this article, unless otherwise stated in a credit line to the data. 
pathways $[9,10]$. Necrostatin-1, a cellular catabolic mediator in chondrocytes, also plays a role in OA: it upregulates matrix-degrading enzymes induced by IL-1 $\beta$ [11].

Arginine methylation is a post-translational modification commonly found in mammalian cells and catalyzed by proteins of the arginine methyltransferase (PRMT) family [12, 13]. PRMT5, a type II enzyme, is found in both the cytoplasm and the nucleus of cells $[14,15]$; it is involved in many physiological processes, including adipogenesis [16], hematopoiesis [17], and spermatogenesis $[18,19]$. The overexpression of PRMT5 in various tumors and in leukemia has also been reported [19-21]. Recent studies indicate that PRMT5 participates in the maintenance of chondrogenic progenitor cells in the limb bud [22]. It is also involved in inflammation, migration of fibroblast-like synoviocytes in rheumatoid arthritis, and induction of an inflammatory reaction in the endothelium $[23,24]$. In addition, several studies have shown that PRMT5 regulates the NF- $\mathrm{KB}$ pathway [25-28], both via TRAIL [25] and by dimethylation of R30 on the p65 subunit [26].

Here, we report the specific upregulation of PRMT5 in human OA cartilage and show that the inhibition of PRMT5 expression attenuates IL-1 $\beta$-mediated MMP-3 and MMP-13 expression levels through activation of the MAPK and NF- $\mathrm{KB}$ signaling pathways of chondrocytes. These results imply that PRMT5 acts as a catabolic regulator in the pathogenesis of OA.

\section{Materials and methods}

\section{Clinical sample collection}

Clinical specimens (cartilage) were obtained from three patients with OA (two women and one man; age 67.1 \pm 8.4 years, range $58-75$ years) who underwent total knee arthroplasty. The three patients in the control group (two women and one man; age $30.1 \pm 5.6$ years, range 18-42 years) underwent total knee arthroplasty related to osteosarcoma involving the knee joint patients; they had no history of OA. All surgeries were performed at the People's Hospital of Zhengzhou University (Zhengzhou, China). The study was approved by the hospital's Clinical Research Ethics Committee (approval no. 2017237), and informed consent was obtained from each tissue donor prior to inclusion in this study.

\section{Animal models}

Male C57BL/6 mice (12 weeks old and weighing 20-25 g) used in this study were housed at the Experimental Animal Center of the People's Hospital of Zhengzhou University and fed a standard diet under specific pathogen-free conditions. An OA model was established in the mice by destabilized medial meniscus (DMM) surgery of the right knee joint, in accordance with a previously described protocol [29]. The 30 mice were randomly divided into three groups of 10 mice each: (1) in the sham group, sham-operated mice were administered vehicle (dimethylsulfoxide); (2) in the DMM group, the mice underwent DMM surgery and were administered vehicle; (3) in the DMM+EPZ group, the mice underwent DMM surgery and were administered the PRMT5 inhibitor EPZ $(200 \mu \mathrm{g} / \mathrm{kg})$. Vehicle or EPZ solution was injected intra-articularly, twice per week for 8 weeks.

Approval for the animal experiments was obtained from the Institutional Animal Care and Use Committee (IACUC) at the People's Hospital of Zhengzhou University (ethical approval code: 2018526).

\section{Micro-computed tomography imaging}

All mouse $(n=10)$ knee joints were scanned at $100 \mathrm{kV}$ and $98 \mu \mathrm{A}$ using microcomputed tomography $(\mu \mathrm{CT})(\mu-$ CT50 Scanco Medical, Bassersdorf, Switzerland). The resolution was set to $10.5 \mu \mathrm{M}$. Image reconstruction and analysis were performed using the built-in software; these were followed by three-dimensional reconstruction and structural parameter analysis. The structural parameter analysis included determinations of bone volume/tissue volume, trabecular thickness, and trabecular separation.

\section{Histological and immunochemical analyses}

Human cartilage and mouse joint tissue samples were collected and fixed in 4\% formalin, then incubated for 3 weeks in $10 \%$ EDTA and embedded in paraffin. The tissues were cut into $4-\mu \mathrm{m}$ coronal sections for hematoxylin and eosin and safranin O-Fast green staining, as well as immunohistochemistry. For cartilage analysis and scoring, safranin O-Fast green staining was performed, according to the OARSI histologic scoring system [29, 30]. Briefly, the extent of the surface damage was scored in a blinded manner at the mouse tibia (lateral tibia (LT) and medial tibia (MT)) as well as femur (lateral femur (LF) and medial femur (MF)); the score for cartilage damage was calculated in a scale of 0 (normal) to 6 (bone loss, remodeling, deformation), based on the OA depth into the cartilage. The OARSI score was calculated based on averaging of maximum score among the four articular surfaces from ten mice.

Immunohistochemistry staining was performed using the DAB tissue staining SP-kit, in accordance with the standard protocol. Tissue sections were incubated overnight with specific anti-MMP-13, MMP-3, PRMT5, pp38, and p-p65 antibodies at $4{ }^{\circ} \mathrm{C}$ and then analyzed by optical microscopy.

\section{Cell experiments}

Cartilage samples collected from patients with OA during total knee arthroplasty were washed, cut into pieces in $4{ }^{\circ} \mathrm{C}$ sterile phosphate buffer, and digested with $0.25 \%$ 
trypsin at $37^{\circ} \mathrm{C}$ for $30 \mathrm{~min}$; they were then incubated in $0.25 \%$ collagenase II at $37^{\circ} \mathrm{C}$ for $24 \mathrm{~h}$. The chondrocytes were cultured in DMEM/F12 with 10\% fetal bovine serum, $100 \mathrm{U} / \mathrm{mL}$ penicillin, and $100 \mathrm{mg} / \mathrm{mL}$ streptomycin at $37^{\circ} \mathrm{C}$ under $5 \% \mathrm{CO}_{2}$. Chondrocytes were passaged twice before use in experiments. To study the induction of inflammation, passage 3 chondrocytes were seeded on six-well plates $\left(2 \times 10^{5}\right.$ cells/well $)$ and treated with $10 \mathrm{ng} / \mathrm{mL}$ IL- $1 \beta$ for $72 \mathrm{~h}$ (Sigma-Aldrich, St. Louis, MO, USA). Levels of PRMT5, MMP-3, MMP-13, Collagen II, Sox9, and ADAMTs5 protein were then determined. To investigate the mechanism by which PRMT5 damages articular chondrocytes, we examined the expression of p38, JNK, ERK1/2, p65, IкB, p-JNK, p-p38, p-ERK1/2, p-IкB, and p-p65 in chondrocytes that had been stimulated with IL- $1 \beta$ for 0,15 , and $30 \mathrm{~min}$.

\section{Micromass cultures}

Micromass cultures were performed as previously described [31]. Briefly, chondrocytes were plated at a density of $2.5 \times 10^{5}$ cells $/ 10-\mu \mathrm{L}$ drop. The cell culture medium was replaced with fresh culture medium every other day. On days 5 and 7 , cartilage differentiation was determined by alcian blue staining; the cartilage was then extracted using $6 \mathrm{M}$ guanidine hydrochloride. The absorbance of the supernatant at $600 \mathrm{~nm}$ was measured using a multimode microplate reader (BioTek, Winooski, VT, USA).

\section{Adenovirus vector infection}

Human PRMT5 cDNA was cloned and inserted into a pHBAd-MCMV-GFP expression vector (Wuhan Miaoling Biotechnology Co. Ltd., Hubei, China). Empty vectors were used as controls. Human chondrocytes were cultured in DMEM/F-12 medium without fetal bovine serum in 12 -well plates. When the cultures had reached $70 \%$ confluency, they were transduced with pHBAd-MCMV-GFPPRMT5 (ad-PRMT5) and the vector control (ad-NC), in accordance with the manufacturer's instructions. After the direct addition of recombinant adenoviruses to the medium, the transduced cells were incubated for $4 \mathrm{~h}$; the medium was then changed to vector-free medium. Samples were collected for the measurement of protein and mRNA expression, after the virus vector infection $72 \mathrm{~h}$.

\section{RNA extraction and quantitative real-time PCR}

Total RNA was extracted from human chondrocytes using TRIzol reagent (Invitrogen, Carlsbad, CA, USA), in accordance with the manufacturer's instructions.

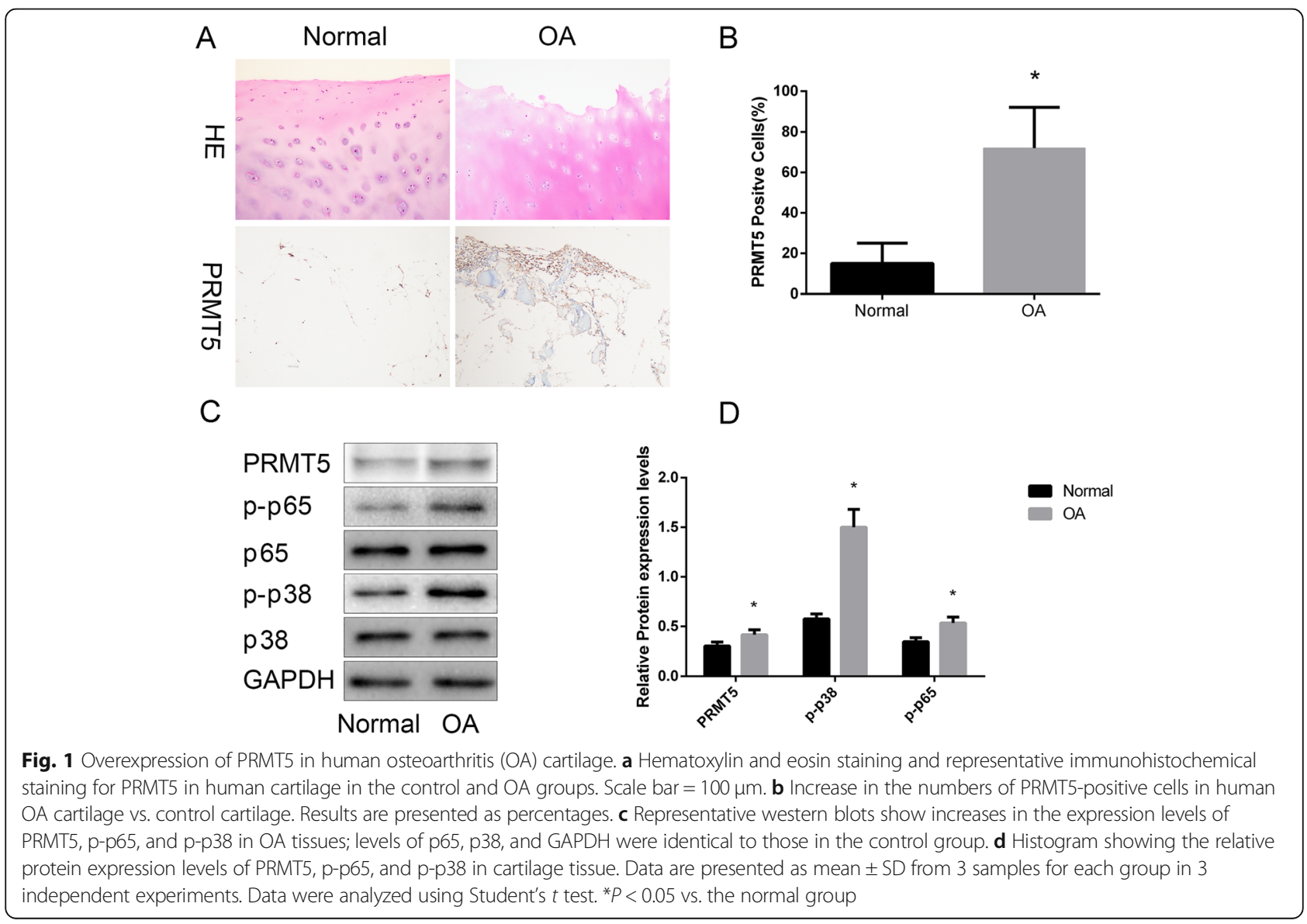


cDNA of each sample was synthesized from $1 \mu \mathrm{g}$ of total RNA using the Revert Aid First Strand cDNA Synthesis Kit (Thermo Scientific, Waltham, MA, USA). The mRNA expression levels of PRMT5, MMP-3, MMP-13, Collagen II, Sox9, and ADAMTs5 were measured by using the qRT-PCR method, and the relative primers are listed in Table S1. Target mRNA expression levels were normalized against GAPDH. The relative expression levels were computed using the $2^{-\Delta \Delta \mathrm{Ct}}$ method.

\section{Western blotting}

The total protein of cartilage samples from OA patients was extracted using tissue homogenizer, and then RIPA buffer supplemented with protease and phosphatase inhibitor (Boster BIO, Wuhan, China) was added and ultimately the cell lysates were centrifuged at $16000 \times g$ for $20 \mathrm{~min}$ to remove the tissue. Protein concentrations of the samples were determined using a BCA protein assay kit (Boster BIO, Wuhan, China). Proteins were extracted from cells using $100 \mu \mathrm{L}$ of RIPA buffer supplemented with protease and phosphatase inhibitor. Lysates were centrifuged for $20 \mathrm{~min}$ at $10,000 \mathrm{~g} ; 20 \mu \mathrm{g}$ of total cellular protein per sample was loaded on a $10 \%$ sodium dodecyl sulfate-polyacrylamide gel, in accordance with the manufacturer's protocol (Boster Bio, Wuhan, China). Protein samples were then transferred to the PVDF membranes (Millipore, Billerica, MA, USA) and blocked for $1 \mathrm{~h}$ with $5 \%$ skim milk in Tris-buffered saline with $0.1 \%$ Tween-20. The blots were then probed overnight at $4{ }^{\circ} \mathrm{C}$ with rabbit primary antibodies against PRMT5,

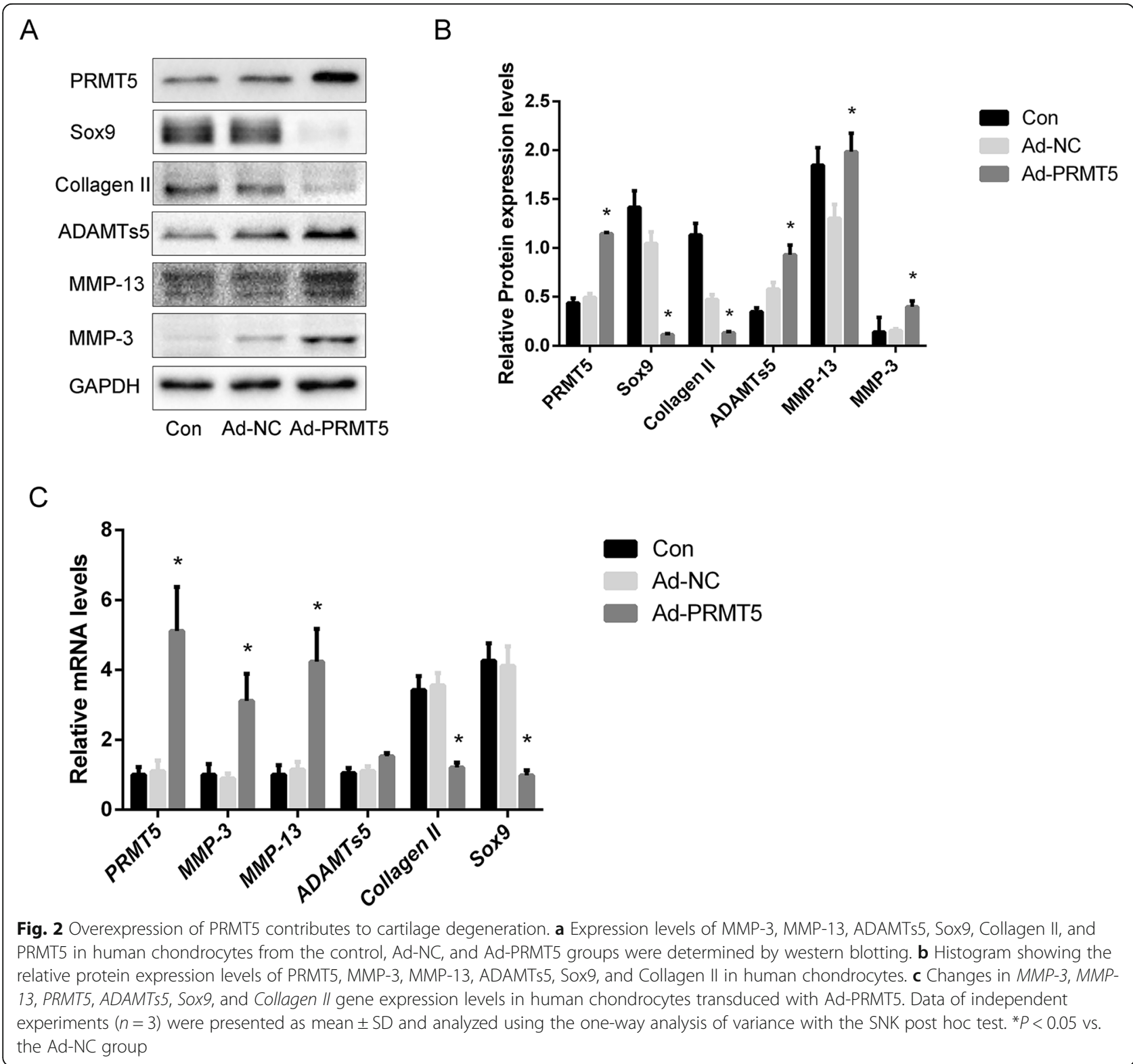


MMP-3, MMP-13, Collagen II, Sox9, ADAMTs5, p38, JNK, ERK1/2, p65, ІкB, p-JNK, p-p38, p-ERK1/2, p-IкB, p-p65, and GAPDH. After the blots were washed three times with Tris-buffered saline with $0.1 \%$ Tween-20, they were incubated with anti-mouse or anti-rabbit secondary antibodies. Immunoreactivity was detected with enhanced chemiluminescence (Bio-Rad Laboratories, Munich, Germany).

\section{Statistical analysis}

The data are presented as means \pm standard deviations of at least three independent experiments. Student's $t$ tests were used to analyze the differences between the two groups, and one-way analysis of variance with the SNK post hoc test was used to compare the differences among the three groups. A two-tailed $p$ value of $<0.05$ was considered to indicate statistical significance.

\section{Results}

PRMT5 is upregulated in OA chondrocytes

To study the role of PRMTT5 in the pathogenesis of OA, we measured the expression of PRMTT5 in the cartilage from OA patients or normal individuals using immunohistochemistry. The results showed that PRMT5 protein levels were markedly elevated in OA human cartilage, compared with the control group cartilage (Fig. 1a, b). Furthermore, western blotting showed upregulation of PRMT5, p-p38, and p-p65 expressions (Fig. 1c), which indicated the activation of NF- $\mathrm{KB}$ and MAPK subtypes in OA human cartilage.

\section{PRMT5 overexpression causes cartilage degeneration}

The role of PRMT5 in the regulation of cartilage metabolism was investigated by the transduction of primary culture human chondrocytes with a PRMT5 adenovirus vector, which resulted in the overexpression of the protein. As shown in Fig. 2a, compared with the control group, PRMT5 overexpression induced the upregulation of MMP-3, MMP-13, and ADAMTs5, as well as the downregulation of Collagen II and Sox9. Elevated PRMT5 levels also caused the upregulation of $M M P-3$, $M M P-13$, and $A D A M T s 5$ mRNA expression, whereas

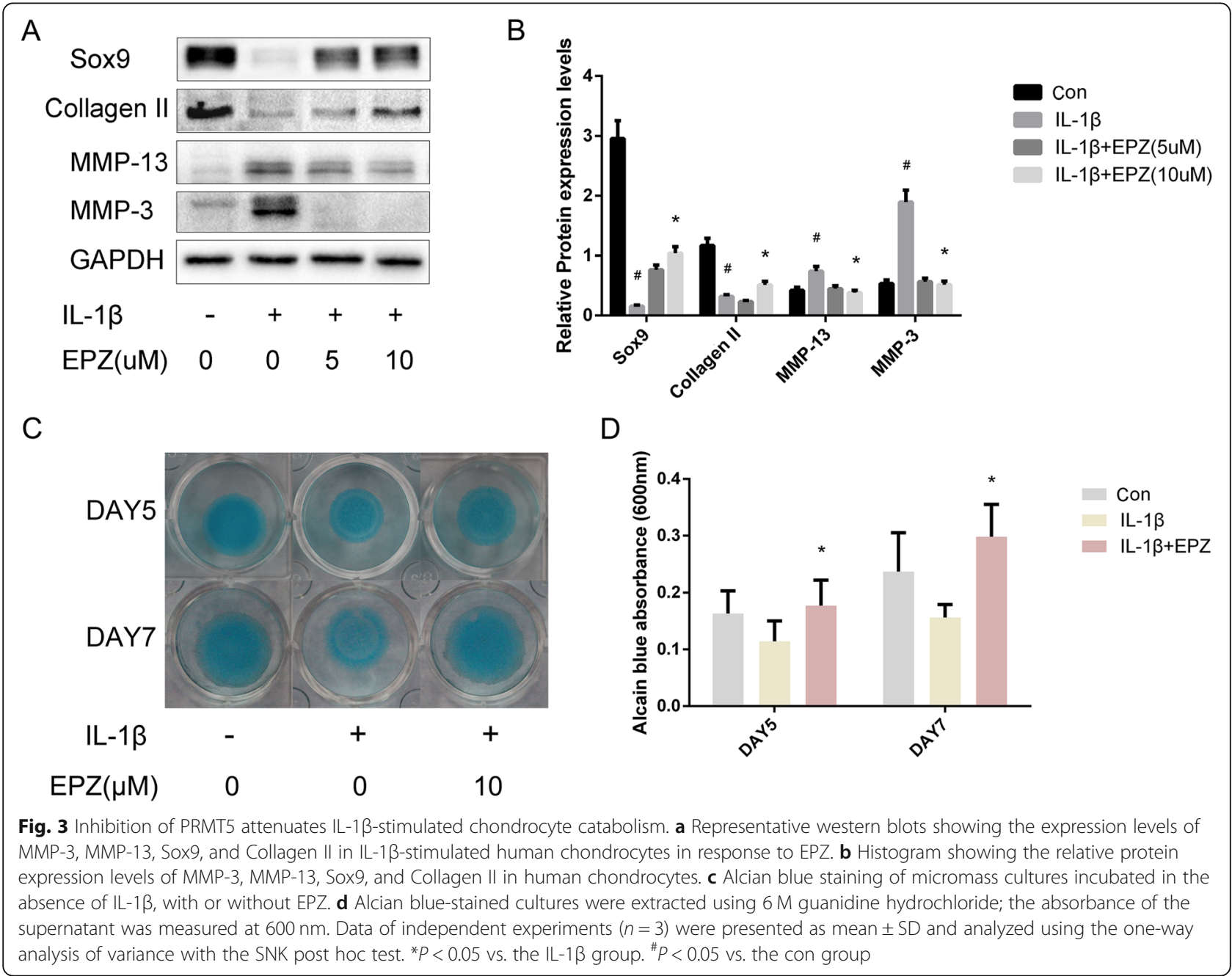


the downregulation of Collagen II and Sox9 mRNA expression was observed (Fig. 2c).

\section{PRMT5 inhibition attenuates IL-1 $\beta$-stimulated induced chondrocyte catabolism}

To determine the effect of PRMT5 inhibition on IL-1 $\beta$ stimulated chondrocyte degeneration, primary cultures of human chondrocytes were co-treated with EPZ in the presence or absence of IL-1 $\beta$ for $72 \mathrm{~h}$. As shown in Fig. 3a, MMP-3 and MMP-13 protein expression were upregulated, while Collagen II and Sox9 protein expression were downregulated in IL-1 $\beta$-treated cells; in cells

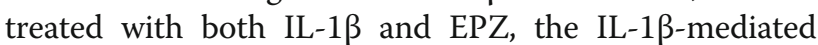

responses were attenuated. The ability of EPZ to affect early chondrogenesis by primary culture human chondrocytes stimulated with IL- $1 \beta$ for 5 and 7 days was determined by using in vitro high-density micromass cultures. Figure $3 c, d$ also shows that IL- $1 \beta$ reduced the alcian blue incorporation, an effect counteracted by the presence of EPZ.

\section{PRMT5 inhibition confers protection against experimental OA}

The effect of PRMT5 inhibition on experimental OA pathogenesis was assessed in a DMM mouse model. DMM results in cartilage erosion, osteophyte formation,

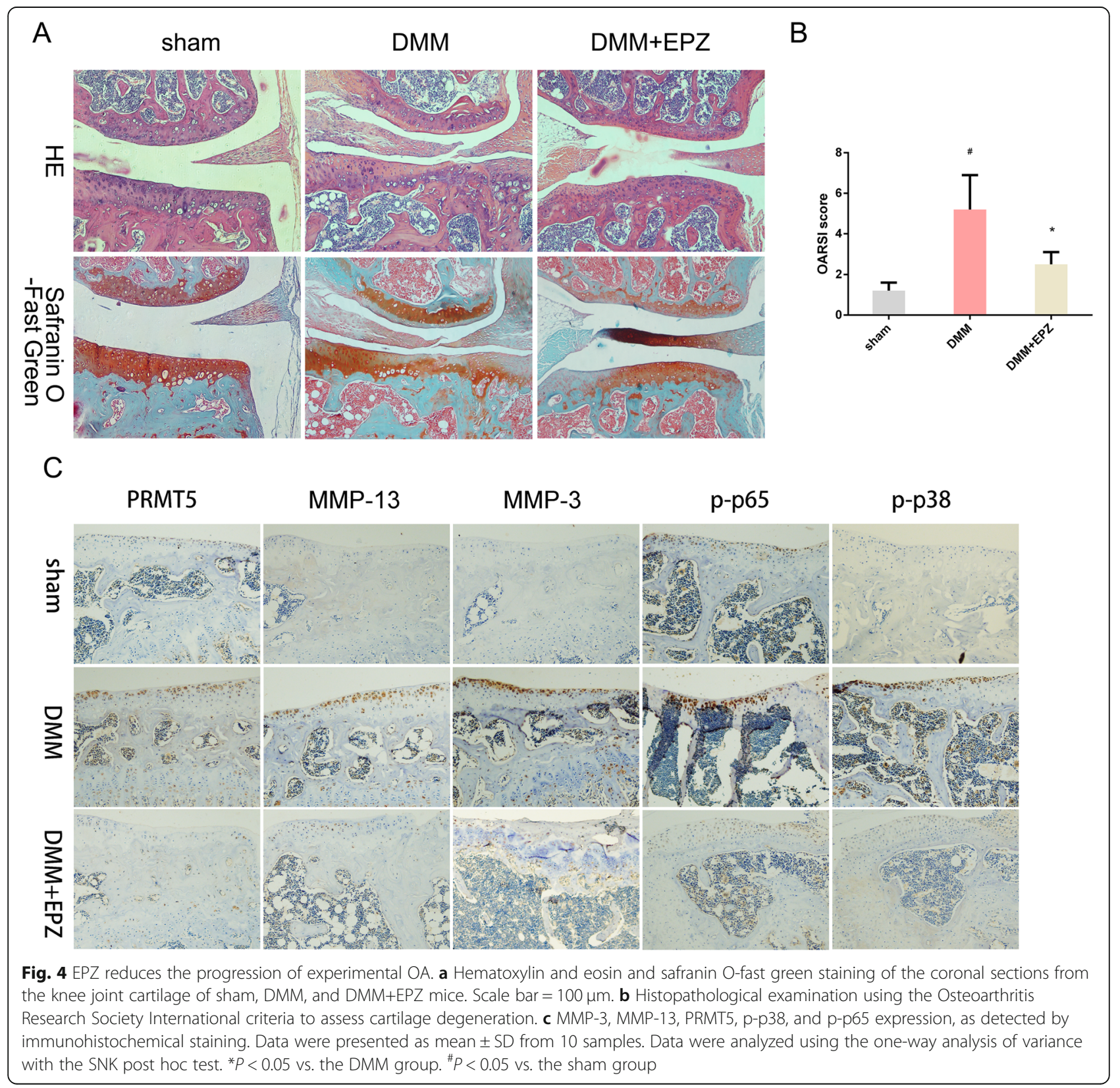


and subchondral bone plate thickening [32, 33]; therefore, it serves as a model of OA. Figure 4a shows that EPZ significantly inhibited cartilage destruction in DMM mice; it also reduced the DMM-induced upregulation of MMP-3, MMP-13, and PRMT5 expression, whereas the downregulation of p-p38 and p-p65 expression were observed in the $\mathrm{DMM}+\mathrm{EPZ}$ group, implying that EPZ can neutralize the activation of the NF-kB and MAPK pathways induced by DMM (Fig. 4c). The Osteoarthritis Research Society International score indicated a protective effect of EPZ on the articular cartilage (Fig. 4a-c). Dramatic changes in total subchondral bone tissue were revealed by $\mu \mathrm{CT}$, with $47.5 \%$ and $36.2 \%$ reductions in bone volume/tissue volume and trabecular thickness, respectively, in DMM mice compared to sham-operated mice. Furthermore, trabecular separation increased by $16.8 \%$ in DMM mice, whereas EPZ attenuated tibial subchondral bone loss, compared to vehicle-treated DMM mice (Fig. 5).

NF-KB and MAPK mediate PRMT5 activity in chondrocytes To investigate the mechanism by which PRMT5 damages articular chondrocytes, we examined the activation statuses of the NF- $\mathrm{KB}$ and MAPK pathways in chondrocytes that had been stimulated with IL-1 $\beta$ in the presence or absence of EPZ. Figure 6 shows that EPZ inhibited the upregulation of phosphorylated-IкB $\alpha$, p65, p38, and JNK, which had been induced by IL-1 $\beta$. Thus, the effects of EPZ on IL-1 $\beta$-induced PRMT5 included inhibition of the NF- $\mathrm{kB}$ and MAPK signaling pathways.

\section{Discussion}

There remains no effective treatment for OA, and the mechanisms underlying its pathogenesis are poorly understood [34]. PRMT5 is abundantly expressed in a wide variety of human cells [35], and its expression is associated with the occurrence and progression of various diseases [36]; however, its role in the development and progression of OA has been unclear. Our study showed that PRMT5 contributes to OA pathogenesis in mice by modulating the expression levels of MMP-3 and MMP13 in chondrocytes; in addition, PRMT5 is upregulated in IL-1 $\beta$-stimulated chondrocytes of patients with OA. The ability of PRMT5 to increase the levels of catabolic

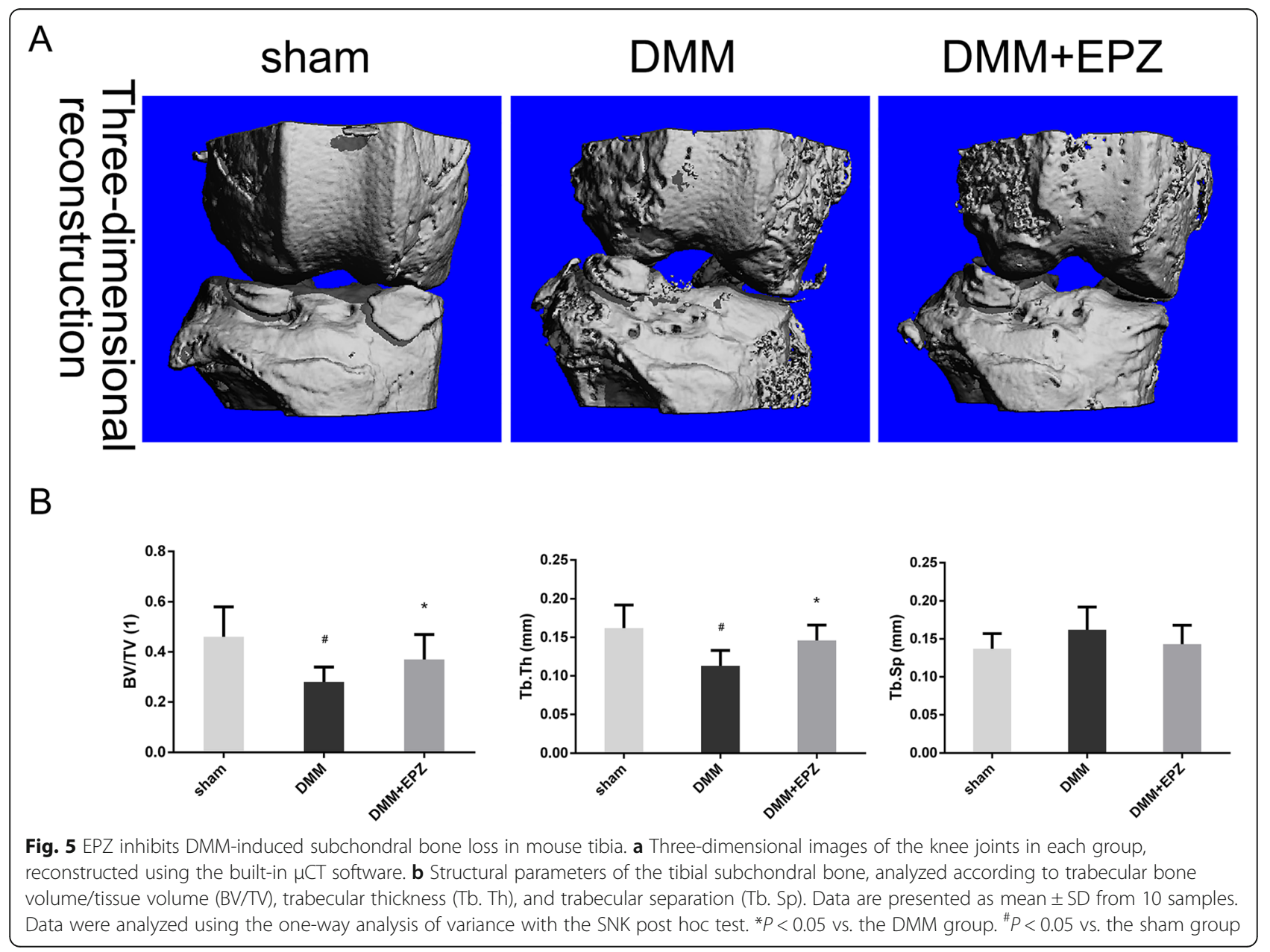




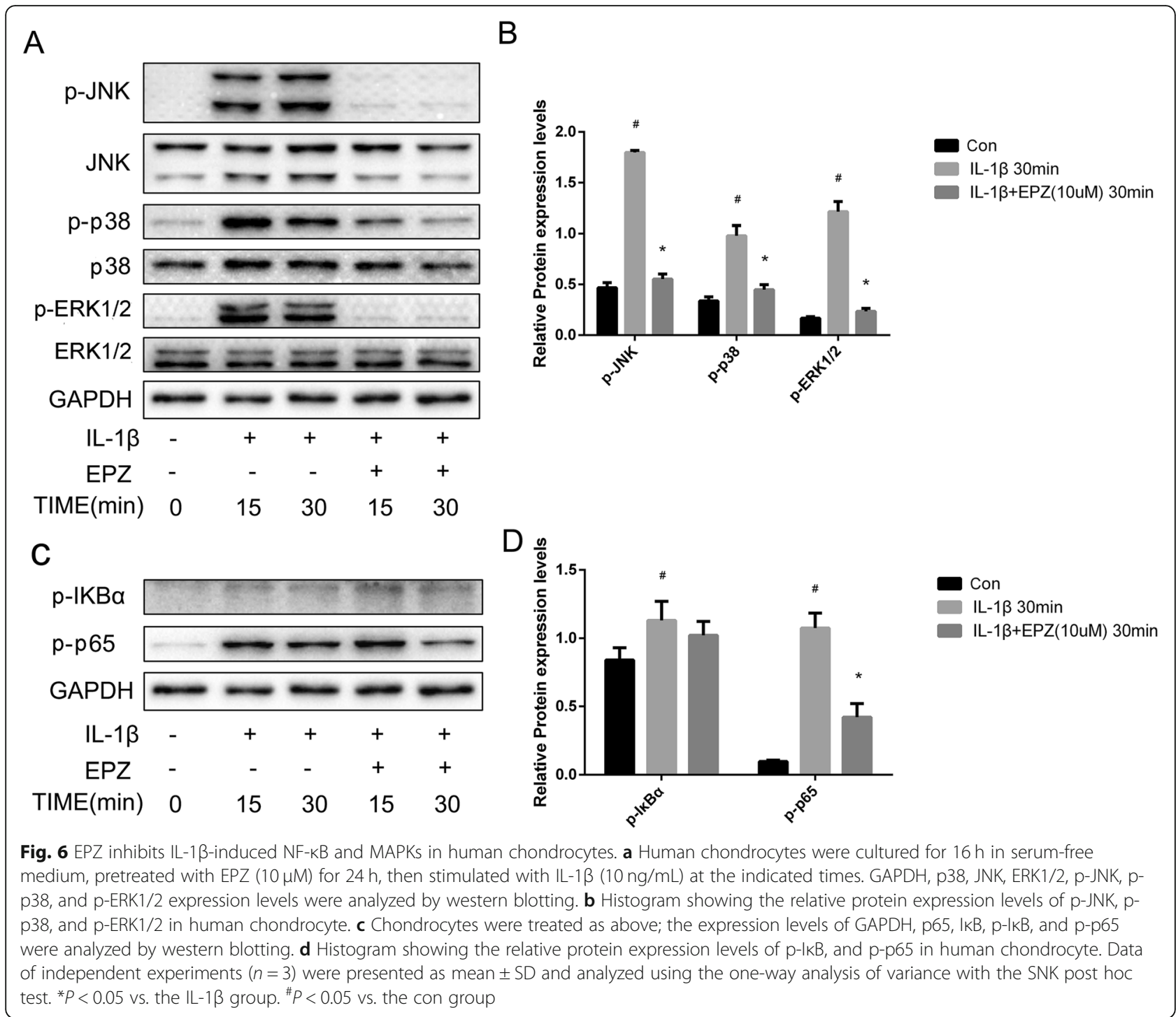

factors suggests that it is a crucial catabolic regulator involved in OA.

Increasing evidences suggest that $\mathrm{OA}$ is a disease of the whole joint including the cartilage, synovium, ligaments, bone, and bone marrow. In recent years, the subchondral bone has been considered to be one of the main pathological features of OA [37-39]. In OA, there is a significant loss of trabecular bone from the tibial subchondral bone. Together with bone marrow lesions, this bone loss is closely linked to pain and is predictive of the severity of cartilage damage in OA. Osteoclasts play a role in the remodeling of the subchondral bone. In this study, we found that EPZ can inhibit DMMinduced subchondral bone loss. These study results were consistent with a previous study which showed that subchondral bone loss is in the DMM OA model [40]. On the contrary, some studies had found that trabecular bone volume/tissue volume (BV/TV) and tibial thickness increased in the subchondral bone of the DMM model $[41,42]$. PRMT5 has been demonstrated to regulate osteoclast differentiation and protect against the bonerelated effects of ovariectomy in our previous study [43]. However, this study revealed that EPZ by intraarticular injection could not affect the activation of osteoclast in the subchondral bone (Fig. S1). Evidences suggest that cross-talk between chondrocytes and subchondral bone may be involved in the pathology of OA [44, 45]. Therefore, we conferred that EPZ that mainly affected the metabolism of chondrocytes involved in the development of OA may be plausibility. NF- $\mathrm{kB}$ comprises a family of ubiquitously expressed transcription factors involved in immunity, stress responses (including responses to mechanical stress), and inflammatory diseases [46]. Our results indicated that the upregulation of MMP-3 and MMP-13 by IL$1 \beta$-induced PRMT5 during cartilage destruction is 
mediated by NF-KB. However, because the effect was only partially blocked by EPZ, other PRMT5-inducing signaling mechanisms may also be involved.

Ramachandran et al. showed that chondrocytes without PRMT5 were unable to undergo hypertrophic differentiation [47], a process related to articular cartilage degeneration. Dongying Chen et al. demonstrated the important roles of PRMT5 in inflammatory responses, cell proliferation, and migration and invasion of fibroblast-like synoviocytes in rheumatoid arthritis; notably, they showed that these responses are mediated by the NF- $\mathrm{kB}$ and AKT pathways [24]. Taken together, these results implicate PRMT5 in synovial lesions of OA. In contrast, Sun and colleagues showed that PRMT5 promotes the expression of Sox9 to maintain type collagen II expression levels in human juvenile costal chondrocytes [48]. The discrepancies among studies may be related to the different cell lines used in the respective experiments.

A limitation of our study was that only one time point was examined in the DMM mouse experiment, which evaluated the effect of PRMT5 on OA progression. In addition, while our results implicate the NF- $\mathrm{kB}$ pathway in PRMT5-mediated OA pathology, the specific mechanism linking the molecular events to cartilage damage remains unknown. Future research should involve analysis of multiple time points in animal studies and a larger number of patients in clinical studies to better understand the specific downstream mechanisms of PRMT5 in the development of OA.

\section{Conclusions}

Our study identified PRMT5 as a newly described catabolic regulator of OA pathogenesis. Overexpression of PRMT5 upregulated the expression levels of matrixdegrading enzymes in chondrocytes of patients with OA, an effect that could be partially attributed to the activation of the NF- $\mathrm{KB}$ and MAPK signaling pathways. Although the specific mechanism is not yet known, our results suggest that PRMT5 should be further explored for the prevention and treatment of OA.

\section{Supplementary information}

Supplementary information accompanies this paper at https://doi.org/10. 1186/s13075-020-02304-x.

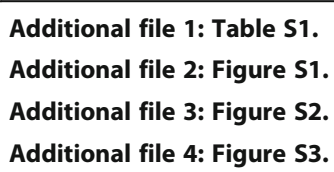

\section{Abbreviations}

DMM: Destabilized medial meniscus; EPZ: EPZ015666; IL: Interleukin MAPK: Mitogen-activated protein kinase; MMP: Matrix metalloproteinase; NFKB: Nuclear factor kappa-B; OA: Osteoarthritis; PRMT5: Protein arginine Nmethyltransferase 5; $\mu \mathrm{CT}$ : Microcomputed tomography

\section{Acknowledgements}

The authors would like to thank all OA and osteosarcoma patients for their participation in the study.

\section{Authors' contributions \\ $J Z$ and YD designed the experiments and revised the manuscript. YD and PW conducted the experiments and prepared the manuscript. YY, JH, ZD, and $W Z$ analyzed the data. $C T, W P$, and $K L$ contributed reagents and materials. $\mathrm{YJ}$ and $\mathrm{HZ}$ assisted with the manuscript revision. All authors have read and approved the final manuscript}

\section{Funding}

This work was supported by the China Postdoctoral Fund Project (2018 M632780), the Ministry of Science and Technology National Key Research and Development Program (122300411149), the Natural Science Foundation of Henan Province (182300410349), and the Joint Construction Project of Henan Provincial Health Committee and Ministry of Health (201701017).

\section{Availability of data and materials}

The datasets used and/or analyzed during the current study are available from the corresponding author on reasonable request.

\section{Ethics approval and consent to participate}

The animal study in this research was approved by the Institutional Animal Care and Use Committee (IACUC) at the People's Hospital of Zhengzhou University (approval no. 2018526). The clinical specimen study was approved by the People's Hospital of Zhengzhou University Clinical Research Ethics Committee (approval no. 2017237), and informed consent was obtained from each donor.

Consent for publication

Not applicable.

\section{Competing interests}

The authors have no competing interests to declare.

\section{Author details}

${ }^{1}$ Department of Orthopedics, Henan Provincial People's Hospital, Zhengzhou University People's Hospital, Henan University People's Hospital, No.7, Weiwu Road, Zhengzhou 450003, Henan Province, China. ${ }^{2}$ Department of pathophysiology, School of Basic Medical Sciences, Zhengzhou University, Zhengzhou 450001, China. ${ }^{3}$ Department of Molecular Pathology, The Affiliated Cancer Hospital of Zhengzhou University, Henan Cancer Hospital, Zhengzhou 450008, China.

Received: 29 February 2020 Accepted: 25 August 2020

Published online: 04 September 2020

\section{References}

1. Hunter DJ, Schofield D, Callander E. The individual and socioeconomic impact of osteoarthritis. Nat Rev Rheumatol. 2014;10:437-41.

2. Hunter DJ, Bierma-Zeinstra S. Osteoarthritis. Lancet. 2019;393:1745-59.

3. Glyn-Jones S, Palmer AJ, Agricola R, Price AJ, Vincent TL, Weinans $H$, et al. Osteoarthritis. Lancet. 2015:386:376-87.

4. Blom $A B$, van Lent $P L$, Libregts $S$, Holthuysen $A E$, van der Kraan $P M$, van Rooijen $\mathrm{N}$, et al. Crucial role of macrophages in matrix metalloproteinasemediated cartilage destruction during experimental osteoarthritis: involvement of matrix metalloproteinase 3. Arthritis Rheum. 2007:56:147-57.

5. Tetlow LC, Adlam DJ, Woolley DE. Matrix metalloproteinase and proinflammatory cytokine production by chondrocytes of human osteoarthritic cartilage: associations with degenerative changes. Arthritis Rheum. 2001:44:585-94.

6. Li H, Wang D, Yuan Y, Min J. New insights on the MMP-13 regulatory network in the pathogenesis of early osteoarthritis. Arthritis Res Ther. 2017; 19:248.

7. Kobayashi M, Squires GR, Mousa A, Tanzer M, Zukor DJ, Antoniou J, et al. Role of interleukin-1 and tumor necrosis factor alpha in matrix degradation of human osteoarthritic cartilage. Arthritis Rheum. 2005;52:128-35.

8. Burrage PS, Mix KS, Brinckerhoff CE. Matrix metalloproteinases: role in arthritis. Front Biosci. 2006;11:529-43. 
9. Shanmugam MK, Ahn KS, Lee JH, Kannaiyan R, Mustafa N, Manu KA, et al. Celastrol attenuates the invasion and migration and augments the anticancer effects of bortezomib in a xenograft mouse model of multiple myeloma. Front Pharmacol. 2018;9:365.

10. Nissinen L, Kahari VM. Matrix metalloproteinases in inflammation. Biochim Biophys Acta. 1840;2014:2571-80.

11. Liang S, Lv ZT, Zhang JM, Wang YT, Dong YH, Wang ZG, et al. Necrostatin-1 attenuates trauma-induced mouse osteoarthritis and IL-1 $\beta$ induced apoptosis via HMGB1/TLR4/SDF-1 in primary mouse chondrocytes. Front Pharmacol. 2018;9:1378.

12. Jansson M, Durant ST, Cho EC, Sheahan S, Edelmann M, Kessler B, et al. Arginine methylation regulates the p53 response. Nat Cell Biol. 2008;10: 1431-9.

13. Krause CD, Yang ZH, Kim YS, Lee JH, Cook JR, Pestka S. Protein arginine methyltransferases: evolution and assessment of their pharmacological and therapeutic potential. Pharmacol Ther. 2007;113:50-87.

14. Banasavadi-Siddegowda YK, Russell L, Frair E, Karkhanis VA, Relation T, Yoo JY et al. PRMT5-PTEN molecular pathway regulates senescence and self-renewal of primary glioblastoma neurosphere cells. Oncogene. 2017;36:263-74.

15. Pasternack DA, Sayegh J, Clarke S, Read LK. Evolutionarily divergent type II protein arginine methyltransferase in Trypanosoma brucei. Eukaryot Cell. 2007;6:1665-81

16. LeBlanc SE, Konda S, Wu Q, Hu YJ, Oslowski CM, Sif S, et al. Protein arginine methyltransferase 5 (Prmt5) promotes gene expression of peroxisome proliferator-activated receptor $\gamma 2$ (PPARy2) and its target genes during adipogenesis. Mol Endocrinol. 2012;26:583-97.

17. Liu L, Zhao X, Zhao L, Li J, Yang H, Zhu Z, et al. Arginine methylation of SREBP1a via PRMT5 promotes de novo lipogenesis and tumor growth. Cancer Res. 2016;76:1260-72.

18. Wang Y, Zhu T, Li Q, Liu C, Han F, Chen M, et al. Prmt5 is required for germ cell survival during spermatogenesis in mice. Sci Rep. 2015;5:11031.

19. Wang $Y$, Hu W, Yuan Y. Protein arginine methyltransferase 5 (PRMT5) as an anticancer target and its inhibitor discovery. J Med Chem. 2018;61:9429-41.

20. Yoshimatsu M, Toyokawa G, Hayami S, Unoki M, Tsunoda T, Field HI, et al. Dysregulation of PRMT1 and PRMT6, type I arginine methyltransferases, is involved in various types of human cancers. Int J Cancer. 2011;128:562-73.

21. Karkhanis $V$, Hu YJ, Baiocchi RA, Imbalzano AN, Sif S. Versatility of PRMT5induced methylation in growth control and development. Trends Biochem Sci. 2011;36:633-41.

22. Norrie JL, Li Q, Co S, Huang BL, Ding D, Uy JC, et al. PRMT5 is essential for the maintenance of chondrogenic progenitor cells in the limb bud. Development. 2016;143:4608-19.

23. Bandyopadhyay S, Harris DP, Adams GN, Lause GE, McHugh A, Tillmaand EG, et al. HOXA9 methylation by PRMT5 is essential for endothelial cell expression of leukocyte adhesion molecules. Mol Cell Biol. 2012;32:1202-13.

24. Chen D, Zeng S, Huang M, Xu H, Liang L, Yang X. Role of protein arginine methyltransferase 5 in inflammation and migration of fibroblast-like synoviocytes in rheumatoid arthritis. J Cell Mol Med. 2017;21:781-90.

25. Tanaka H, Hoshikawa Y, Oh-hara T, Koike S, Naito M, Noda T, et al. PRMT5, a novel TRAIL receptor-binding protein, inhibits TRAlL-induced apoptosis via nuclear factor-kappaB activation. Mol Cancer Res. 2009;7:557-69.

26. Wei H, Wang B, Miyagi M, She Y, Gopalan B, Huang DB, et al. PRMT5 dimethylates R30 of the p65 subunit to activate NF-kB. Proc Natl Acad Sci U S A. 2013;110:13516-21.

27. Gulla A, Hideshima T, Bianchi G, Fulciniti M, Kemal Samur M, Qi J, et al. Protein arginine methyltransferase 5 has prognostic relevance and is a druggable target in multiple myeloma. Leukemia. 2018;32:996-1002.

28. Harris DP, Chandrasekharan UM, Bandyopadhyay S, Willard B, DiCorleto PE. PRMT5-mediated methylation of NF-KB p65 at Arg174 is required for endothelial CXCL11 gene induction in response to TNF- $a$ and IFN- $\gamma$ costimulation. PLoS One. 2016;11:e0148905.

29. Glasson SS, Blanchet TJ, Morris EA. The surgical destabilization of the medial meniscus (DMM) model of osteoarthritis in the 129/SvEv mouse. Osteoarthr Cartil. 2007;15:1061-9.

30. Glasson SS, Chambers MG, Van Den Berg WB and Little CB. The OARSI histopathology initiative recommendations for histological assessments of osteoarthritis in the mouse. Osteoarthritis Cartilage. 2010;null (undefined): S17-23.

31. Kim GW, Han MS, Park HR, Lee EJ, Jung YK, Usmani SE, et al. CXC chemokine ligand 12a enhances chondrocyte proliferation and maturation during endochondral bone formation. Osteoarthr Cartil. 2015;23:966-74.
32. Fang H, Huang L, Welch I, Norley C, Holdsworth DW, Beier F, et al. Early changes of articular cartilage and subchondral bone in the DMM mouse model of osteoarthritis. Sci Rep. 2018;8:2855.

33. Okura T, Matsushita M, Mishima $K$, Esaki R, Seki T, Ishiguro N, et al. Activated FGFR3 prevents subchondral bone sclerosis during the development of osteoarthritis in transgenic mice with achondroplasia. J Orthop Res. 2018;36:300-8.

34. Kloppenburg M, Berenbaum F. Osteoarthritis year in review 2019: epidemiology and therapy. Osteoarthr Cartil. 2020;28:242-8.

35. Onikubo T, Nicklay JJ, Xing L, Warren C, Anson B, Wang WL, et al. Developmentally regulated post-translational modification of nucleoplasmin controls histone sequestration and deposition. Cell Rep. 2015;10:1735-48.

36. Blanc RS, Richard S. Arginine methylation: the coming of age. Mol Cell. 2017;65:8-24.

37. Inhibition of SDF-1a/CXCR4 signalling in subchondral bone attenuates posttraumatic osteoarthritis. Int J Mol Sci. 2016:17:943.

38. Chen Y, Zhang D, Ho KW, Lin S, Suen WC, Zhang H, et al. GPR120 is an important inflammatory regulator in the development of osteoarthritis. Arthritis Res Ther. 2018;20:163.

39. Findlay DM, Kuliwaba JS. Bone-cartilage crosstalk: a conversation for understanding osteoarthritis. Bone Res. 2016;4:16028.

40. Pang C, Wen L, Qin H, Zhu B, Lu X, Luo S. Sotrastaurin, a PKC inhibitor, attenuates RANKL-induced bone resorption and attenuates osteochondral pathologies associated with the development of OA. J Cell Mol Med. 2020; 24:8452-65.

41. Wang TR, Wang HD, Chen W, Yu TB, Qin Y, Zhang YZ. Proximal fibular osteotomy alleviates medial compartment knee osteoarthritis in a mouse model. Int Orthop. 2020;44:1107-13.

42. Liu B, Ji C, Shao Y, Liang T, He J, Jiang H, et al. Etoricoxib decreases subchondral bone mass and attenuates biomechanical properties at the early stage of osteoarthritis in a mouse model. Biomed Pharmacother. 2020; 127:110144.

43. Dong Y, Song C, Wang Y, Lei Z, Xu F, Guan H, et al. Inhibition of PRMT5 suppresses osteoclast differentiation and partially protects against ovariectomy-induced bone loss through downregulation of CXCL10 and RSAD2. Cell Signal. 2017:34:55-65.

44. Maas O, Joseph GB, Sommer G, Wild D, Kretzschmar M. Association between cartilage degeneration and subchondral bone remodeling in patients with knee osteoarthritis comparing MRI and (99m) TC-DPD-SPECT/ CT. Osteoarthr Cartil. 2015;23:1713-20.

45. Ni Z, Zhou S, Li S, Kuang L, Chen H, Luo X, et al. Exosomes: roles and therapeutic potential in osteoarthritis. Bone Res. 2020;8:25.

46. Rigoglou S, Papavassiliou AG. The NF-kB signalling pathway in osteoarthritis. Int J Biochem Cell Biol. 2013:45:2580-4.

47. Ramachandran J, Liu Z, Gray RS, Vokes SA. PRMT5 is necessary to form distinct cartilage identities in the knee and long bone. Dev Biol. 2019;456: 154-63.

48. Sun M, Hussain S, Hu Y, Yan J, Min Z, Lan X, et al. Maintenance of SOX9 stability and ECM homeostasis by selenium-sensitive PRMT5 in cartilage. Osteoarthr Cartil. 2019;27:932-44.

\section{Publisher's Note}

Springer Nature remains neutral with regard to jurisdictional claims in published maps and institutional affiliations.

Ready to submit your research? Choose BMC and benefit from:

- fast, convenient online submission

- thorough peer review by experienced researchers in your field

- rapid publication on acceptance

- support for research data, including large and complex data types

- gold Open Access which fosters wider collaboration and increased citations

- maximum visibility for your research: over $100 \mathrm{M}$ website views per year

At $\mathrm{BMC}$, research is always in progress.

Learn more biomedcentral.com/submissions 